\title{
Effects of the brake shoe friction material on the railway wheel damage
}

\author{
Abdelmajid HAMDAOUI ${ }^{1}$, El Houcine JADDI $^{1}$ \\ ${ }^{1}$ Mohammed V University, Mohammadia School of Engineers, ERD3M Laboratory, Morocco
}

\begin{abstract}
The wheels of a railway axle are the most critical components of a train. These wheels are subjected to several forms of deterioration, including wear, which significantly influences the safety of traffic as well as the dynamic stability of railway vehicles and the lifetime of wheelsets. The objective of this work is to compare the effect of two friction materials of brake shoes at the damage and the wear rate of the ER8 steel wheels.
\end{abstract}

\section{Introduction}

En raison de l'interaction avec le rail et les semelles pendant le freinage, les roues ferroviaires subissent l'usure avec des taux variables dépendant de plusieurs paramètres. Le couple de matériaux roue/semelle est un facteur important dans l'endommagement et l'usure des roues [1]. L'évolution de la forme du profil due à l'usure conduit à des variations des performances dynamiques du train. Par conséquent, les profils originaux sont périodiquement rétablis par reprofilage qui fait diminuer le diamètre et par conséquent la durée de vie de la roue.

La prédiction du taux d'usure des roues devient ainsi importante pour une meilleure planification des interventions de maintenance. Ces opérations fondamentales et périodiquement nécessaires, sont assez couteuses en termes de dépenses et d'indisponibilité du matériel roulant; d'où l'intérêt de réduire leur fréquence et d'optimiser ainsi la durée de vie des roues [2].

\section{L'usure des roues d'un essieu ferroviaire}

L'usure peut être définie comme étant la perte de matière solide subie par une surface de frottement. L'usure dépend non seulement de la nature du matériau mais aussi de d'autres éléments tels que la géométrie de contact, la topographie de la surface, le chargement, la lubrification et l'environnement. Les mécanismes qui causent de tels dommages sont généralement compliqués et il est difficile de distinguer les uns des autres. Dans [3], environ 60 termes décrivant le comportement et les mécanismes de l'usure sont répertoriés. Certains des mécanismes les plus importants liés aux roues d'un essieu ferroviaire sont mentionnés ci-dessous [4] :
- Usure abrasive: usure causée par des surfaces rugueuses et dures glissant l'une sur l'autre ou une usure causée par des particules dures piégées entre deux surfaces comme les débris d'oxyde dur.

- Usure adhésive: usure causée par le cisaillement des jonctions formées entre deux surfaces en contact, parfois utilisé comme synonyme d'usure par glissement à sec.

- Usure chimique (usure corrosive): usure causée par la formation d'oxyde ou d'autres composants sur les surfaces en raison de la réaction chimique des surfaces avec l'environnement.

- Usure érosive: usure due au mouvement relatif des surfaces de contact en présence d'un fluide contenant des particules solides entre les surfaces.

- Fatigue des contacts roulants (RCF): causée par des variations de contraintes cycliques conduisant à la fatigue des matériaux. Elle en résulte généralement la formation des fissures de surface et de profondeur, des piqûres et l'écaillage.

Dans les travaux de recherche concernant la modélisation de l'usure des roues ferroviaires, trois approches ont été identifiés :

- $\quad$ Mesures sur le matériel.

- $\quad$ Tests de laboratoire.

- Modèles de prédiction théorique.

Différentes techniques ont été donc utilisées pour étudier l'usure des aciers des roues ferroviaires. Des relevés de mesure sur les roues ont été utilisés pour analyser les causes de l'usure. Une grande quantité de données a également été recueillie à partir d'expériences effectuées sur des pistes de test spécialement conçues. Certains essais en laboratoire ont été réalisés à grand échelle et d'autres à échelle réduite [5]. 
Les travaux de recherche antérieurs effectués pour étudier le comportement d'usure des aciers des roues ferroviaires ont permis d'identifier un certain nombre de régimes d'usure. Les premiers tests ont démontré que deux régimes existaient. Ceux-ci ont été désignés comme légers et sévères. Un troisième régime d'usure qualifié catastrophique a été également identifié [6].

Un essai à deux disques a permis de modéliser trois régimes d'usure pour les roues en acier ER8: Doux, grave et catastrophique. Ceux-ci ont été classés en fonction du taux d'usure [7].

\section{Effet du matériau des semelles de frein sur l'endommagement des roues ferroviaires}

L'action de freinage s'effectue par l'appui des semelles de freins sur la table de roulement de la roue comme indiqué sur la Figure 1.

Les semelles de freins sont généralement fabriquées en fonte, en matériaux composites organiques ou en matériaux frittés. Le développement des matériaux pour les semelles au cours des deux dernières décennies a permis l'introduction de nouveaux matériaux de freinage dans l'industrie ferroviaire.

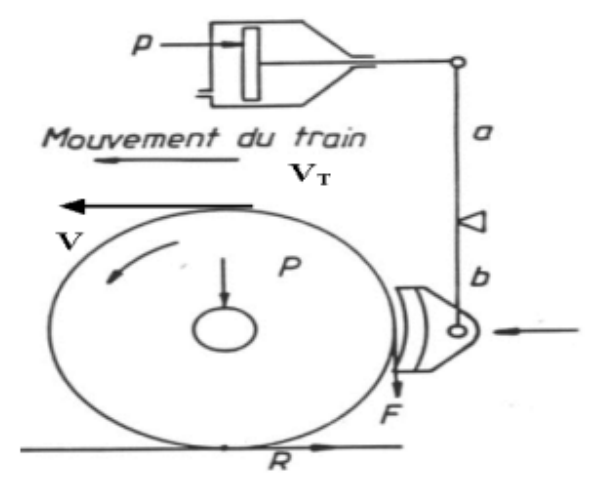

Fig. 1 Freinage par semelles

Des recherches ont été réalisées sur l'influence de divers types de matériaux de semelles sur les performances des freins et les émissions de bruit [8-9]. En conséquence, de nouvelles normes [10] et de nouvelles réglementations pour remplacer les semelles en fonte avec des semelles en matériaux composites sont en cours de développement.

Dans une étude expérimentale récente [11], la comparaison entre les semelles en fonte et les semelles en composite montre que les deux matériaux de semelles testés présentent des différences dans la distribution granulométrique, la morphologie et le contenu élémentaire. Le taux de génération de particules observé est significativement plus important pour la fonte comparée au matériau composite.

Les résultats de d'autres essais confirment une influence significative des semelles en combinaison avec des charges mécaniques sur l'endommagement de la table de roulement [12].

Il a été également prouvé que les semelles en composite produisent des températures plus élevées en par rapport aux semelles en fonte sous les mêmes conditions de freinage. En outre, les semelles en composite imposent moins de dégâts et moins de changement dans les contraintes résiduelles. Tandis que les semelles en fonte de haute teneur en phosphore produisent plus de points chauds sur la table de roulement comparativement aux semelles en fonte ordinaire [13].

Des expériences ont été effectuées par Vernersson pour étudier l'influence de différents types de matériaux en bloc et pour suivre l'élévation de la température (points chauds) dans la bande de roulement [14]. L'étude expérimentale de Vernersson conclut que les semelles en fonte produisent immédiatement des points chauds sur la table de roulement tandis que les semelles en composite nécessitent des vitesses plus élevées et des temps plus importants pour générer ces points chauds. Des essais dynamométriques comparatifs des semelles en fonte et celles en composite montre que les semelles en fonte peuvent augmenter la formation des fissures thermiques dans la table de roulement tandis que les semelles en composite ne changent pas la microstructure de la table de roulement [15].

\section{Usure des roues pour deux couples de matériaux Semelle/Roue :}

\subsection{Matériaux des roues ferroviaires}

Les matériaux les plus utilisés dans la production des roues ferroviaires sont les ER7 et ER8, définis dans la norme EN 13262. Ce sont des aciers à structure perliteferrite. La jante de la roue est généralement durcie suivant le traitement thermique : $860^{\circ} \mathrm{C} / 5 \mathrm{~h} /$ eau et 520 ${ }^{\circ} \mathrm{C} / 5 \mathrm{~h} /$ air.

Le Tableau 1 montre les compositions chimiques de ces aciers, avec les teneuses maximales en différents éléments. 
Tableau 1. Composition chimique des matériaux ER7 et ER8

\begin{tabular}{|c|c|c|c|c|c|c|c|c|c|c|c|}
\hline $\begin{array}{c}\text { Steel } \\
\text { grade }\end{array}$ & $\mathbf{C}$ & $\mathbf{S i}$ & $\mathbf{M n}$ & $\mathbf{P}$ & $\mathbf{S}$ & $\mathbf{C r}$ & $\mathbf{C u}$ & $\mathbf{M o}$ & $\mathbf{N i}$ & $\mathbf{V}$ & $\mathbf{C r + M o + N i}$ \\
\hline ER7 & 0.52 & 0.40 & 0.80 & 0.020 & 0.020 & 0.30 & 0.30 & 0.08 & 0.30 & 0.06 & 0.50 \\
\hline ER8 & 0.56 & 0.40 & 0.80 & 0.020 & 0.020 & 0.30 & 0.30 & 0.08 & 0.30 & 0.06 & 0.50 \\
\hline
\end{tabular}

Le Tableau 2 ci-dessous présente les propriétés mécaniques de base qui devraient être atteintes après application du traitement thermique appliqué et avant l'usinage dans l'état final de production.

Tableau 2. Propriétés mécaniques des matériaux ER7 et ER8

\begin{tabular}{|c|c|c|c|c|c|}
\hline \multirow{2}{*}{$\begin{array}{c}\text { Steel } \\
\text { grade }\end{array}$} & \multicolumn{3}{|c|}{ Wheel rim } & \multicolumn{2}{c|}{ Wheel web } \\
\cline { 2 - 6 } & $\mathrm{ReH}$ & $\mathrm{Rm}$ & $\mathrm{A} 5$ & $\mathrm{DRm}$ & $\mathrm{A} 5$ \\
\cline { 2 - 6 } & {$[\mathrm{MPa}]$} & {$[\mathrm{MPa}]$} & {$[\%]$} & {$[\mathrm{MPa}]$} & {$[\%]$} \\
\hline ER7 & $\geq 520$ & $820-940$ & $\geq 14$ & $\geq 110$ & $\geq 16$ \\
\hline ER8 & $\geq 540$ & $860-980$ & $\geq 13$ & $\geq 120$ & $\geq 16$ \\
\hline
\end{tabular}

\subsection{Comparaison de l'usure des roues en ER8 pour des semelles en fonte et les semelles en composite}

Cette comparaison est effectuée par l'analyse de la variation du diamètre des roues en fonction du parcours réalisé par ces roues durant une période prédéfinie. Le taux d'usure est ainsi défini comme la diminution du diamètre de la roue en $\mathrm{mm}$ par $10000 \mathrm{Km}$ de parcours. Les résultats de cette analyse montrent que le taux d'usure pour les roues équipées de semelles en composite est de $4 \mathrm{~mm}$ par $10000 \mathrm{Km}$ tandis qu'une valeur inférieur de $2 \mathrm{~mm}$ par $10000 \mathrm{~km}$ a été constatée pour les roues équipées de semelles en fonte.

L'écart constaté entre ces taux d'usure est dû d'une part à la différence de leur coefficient de frottement en contact avec l'acier ER8, et d'autre part au nombre de reprofilage subit par chaque type de roue. En effet, la diminution du diamètre est due essentiellement à la diminution du bandage de la roue suite au la reprise du profil conforme d'origine par usinage de la roue. Comparativement aux semelles en fonte, les semelles en composite font évoluer rapidement les cotes caractéristiques du profil, indiqué dans la Figure 2 et Figure 3. , vers des valeurs hors intervalle de tolérance, ce qui impose la reprise du profil original conforme par l'usinage.

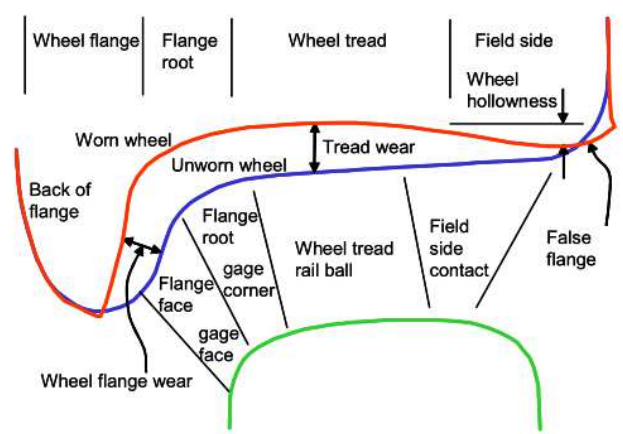

Fig. 2 Evolution du profil par l'usure

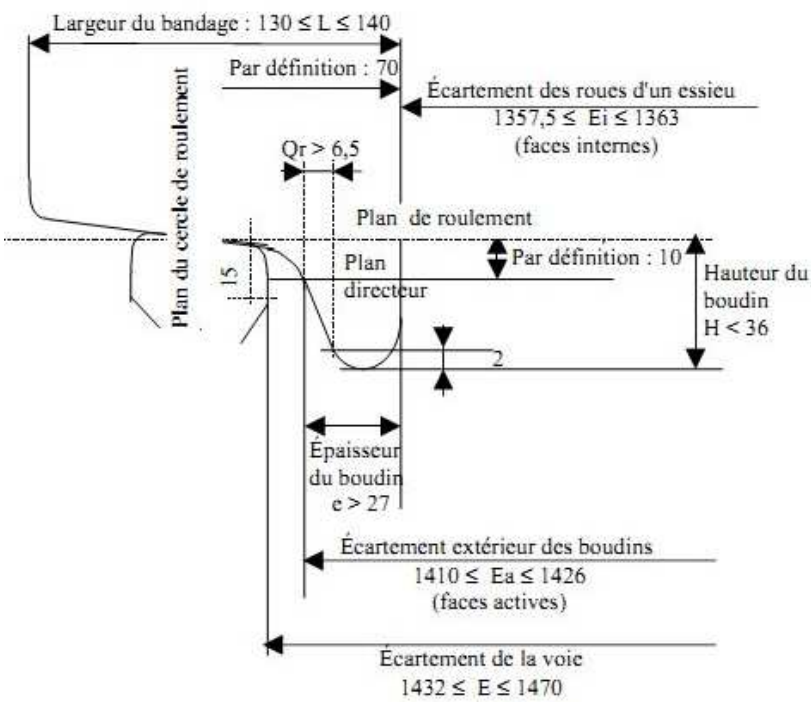

Fig. 3 Cotes caractéristiques du profil de la roue

\section{Conclusion}

Les effets de chaque matériau de semelles sur la dégradation de l'état des roues ferroviaires en service ont été présentés. Malgré les recommandations du passage de la fonte aux matériaux composites pour les semelles de frein en raison de certains avantages acoustiques et environnementaux, l'usage de ces semelles pour des roues en acier ER8 pénalise leur durée de vie et augmente leurs couts de maintenance à cause du taux d'usure important.

\section{References}

1. J. Gerlici, T. Lack, J. Harusinec, Rail vehicles wheels and brake blocks wear laboratory test stand utilization, Scientific journal „Prace Naukowe (Transport: Analiza i ocena elementów systemów transportowych” 101 21-32, (2014).

2. .J. Auciello, M. Ignesti, M. Malvezzi, E. Meli \& A. Rindi Development and validation of a wear model 
for the analysis of the wheel profile evolution in railway vehicles, Vehicle System Dynamics: International Journal of Vehicle Mechanics and Mobility, 50:11, 1707-1734, (2012).

3. P.J. Blau (Ed.), Glossary of terms, Friction, Lubrication, and Wear Technology Metals Handbook, vol. 18, ASME International, Metals Park, OH, (1992).

4. [4]Saeed Hossein Nia, Carlos Casanueva, Sebastian Stichel, Prediction of RCF and wear evolution of iron-ore locomotive wheels, Wear, Volume 338, Pages 62-72, (2015).

5. Kumar, S., Rao, D.L.P. Wheel-rail contact wear, work, and lateral force for zero angle of attack - a laboratory study. Transactions of the ASME, Journal of Dynamic Systems, Measurement, and Control, 106, 319-326, (1984).

6. R. Lewis, U. Olofsson, Mapping rail wear regimes and transitions, Wear, Volume 257, Issue 7, Pages 721-729, (2004).

7. Lewis, R. and Dwyer-Joyce, R.S. Wear mechanisms and transitions in railway wheel steels. Proceedings of the Institution of Mechanical Engineers, Part J: Journal of Engineering Tribology, 218(6), 467-478, (2004)

8. Miyauchi, T., Tsujimura, T., Handa, K., Nakayama, J., and Shimuzu, K. Influence of silicon carbide filters in cast iron composite brake blocks on brake performance and development of a production process. Wear, 267, 833-838, (2009).

9. Bühler, S. Methods and results of field testing of a retrofitted freight train with composite brake blocks. Journal of Sound and Vibration, 293(3-5), 10411050, (2006).

10. Hübner, P. The action programme of UIC, CER and UIP "abatement of railway noise emissions on goods trains". Journal of Sound and Vibration, 231(3), 511-517, (2000).

11. Olofsson, U. A study of airborne wear particles generated from the train traffic-block braking simulation in a pin-on-disc machine. In Proceedings 8th International Conference on Contact Mechanics and Wear of Rail/Wheel Systems, Florence, Italy, pp 527-533, (2009).

12. Stone, D.H. and Carpenter, G.F., Wheel thermal damage limits. Research and Test Department Association of American Railroads: Chicago, IL, USA, pp 57-63, (1994).

13. Stone, D.H. An interpretive review of wheel failure performance with respect to design and heattreatment. In Proceedings Joint ASME/IEEE Railroad Conference, pp 43-53, (1988).

14. Vernersson, T. Thermally induced roughness of tread-braked railway wheels Part 1: brake rig experiments. Wear, 236(1-2), 96-105, (1999).

15. Vukolov, L.A. and Voronchikhin, A.I. New solutions in choice of material and design of brake shoes required by railroad applications. In Proceedings World Tribology Congress III, Washington, D.C., USA, pp 197-198, (2005). 